\title{
The Role of Magnetic Resonance Imaging and the Expression of MMP-9 Protein in the Analysis of Carotid Atherosclerotic Plaques in Patients Undergoing Carotid Endarterectomy: A Prospective Pilot Study
}

\author{
Davorin Sef ${ }^{1}$, Milan Milosevic ${ }^{1}$, Marin Ostric ${ }^{1}$, Tomislav Mestrovic ${ }^{1}$, Bojan Jernej ${ }^{1}$, Slavica \\ Kovacic $^{1}$, Miljenko Kovacevic ${ }^{1}$, Anita Skrtic ${ }^{1}$, and Vinko Vidjak ${ }^{1}$ \\ ${ }^{1}$ Affiliation not available
}

November 1, 2021

\begin{abstract}
Components of carotid atherosclerotic plaque can be analysed preoperatively by non-invasive advanced imaging modalities such as magnetic resonance imaging (MRI). The expression of matrix metalloproteinase-9 protein (MMP-9), which has a potential role in remodelling of atherosclerotic plaques, can be analysed immunohistochemically. The aim of the present prospective pilot study is to analyse histological characteristics and expression of MMP-9 in carotid plaques of patients undergoing carotid endarterectomy (CEA) and to investigate the correlation with preoperative clinical symptoms and MRI features. Preoperative clinical assessment, MRI imaging, postoperative histological and immunohistochemical analyses were performed. Fifteen patients with symptomatic $(7 / 15 ; 47 \%)$ and asymptomatic carotid artery stenosis undergoing CEA were included. Among symptomatic patients, $5(71 \%)$ had recent stroke and $2(29 \%)$ had recent transient ischaemic attack with a median timing of 6 weeks (IQR:1, 18) before the surgery. Both groups did not significantly differ in respect to preoperative characteristics. Prevalence of unstable plaque was higher in symptomatic than asymptomatic patients, although it was not significant (63\% vs. $37 \%, p=0.077)$. The expression of MMP-9 in CD68 cells within the plaque by semiquantitative analysis was found to be significantly higher in symptomatic as compared to asymptomatic patients ( $86 \%$ vs. $25 \%$ with the highest expression, $p=0.014$ ). The average microvascular density was found to be higher and lipid core area larger among both symptomatic patients and unstable carotid plaque specimens, although this did not reach statistical significance $(p=0.064$ and $p=0.132, p=0.360$ and $p=0.569$, respectively). Our results demonstrate that MRI is reliable in classifying carotid lesions and differentiating unstable from stable plaques. We have also shown that the expression of MMP-9 is significantly higher among symptomatic patients undergoing CEA.
\end{abstract}

Trial Registration: This study has been registered at the ISRCTN registry (ID ISRCTN46536832), isrctn.org Identifier: https://www.isrctn.com/ISRCTN46536832

Keywords: atherosclerosis, carotid endarterectomy, unstable plaque, magnetic resonance imaging, matrix metalloproteinase.

\section{Introduction}

Carotid artery disease is the principal cause of ischemic stroke and the benefit of carotid surgical revascularization in both symptomatic and asymptomatic patients has been demonstrated by several trials [1-4]. Atherosclerotic plaque formation is associated with endothelial cells damage, which leads to lipid accumulation, immigration of monocyte-derived macrophages, release of growth factors and activation of vascular smooth muscle cells (VSMC) [5]. Atherosclerotic plaque rupture leads to local platelet activation and thrombosis that may result in embolization and the onset of clinical symptoms [6]. Accumulating evidence confirm the key role of matrix metalloproteinase (MMP) in plaque development and pathogenesis of atherosclerosis [7-9]. MMP, among which is MMP-9, are enzymes that are produced by infiltrating macrophages and VSMC 
and may have an important role in plaque remodelling and destabilization by promoting the degradation of extracellular matrix [8-10]. Increased serum levels of MMP-9 have been detected in the coronary circulation among patients with acute coronary syndrome, which suggested active process of plaque rupture and increased risk of cardiovascular adverse events [11-13]. Similarly, it was found that serum level of MMP-9 was significantly higher in patients with carotid artery stenosis and unstable carotid plaques undergoing carotid endarterectomy (CEA) $[8,14]$. However,Baroncini et al. reported that MMP-9 is a part of atherogenesis process but may not be a factor associated with acute disruption events [15, 16].

Several non-invasive imaging techniques have been evaluated for the characterization of the carotid plaque because carotid angiography is able to detect plaque ulceration but is unable to demonstrate plaque morphology [17]. Despite the fact that duplex ultrasound is the first-line imaging modality, magnetic resonance imaging (MRI) has the advantage to image the aortic arch with supra-aortic arteries as well as to detect the presence of specific plaque features (vulnerable plaque, intra-plaque haemorrhage, lipid-necrotic core) suggesting higher stroke risk [18-22]. Recent reports suggested that preoperative MRI may be useful in the detection of the vulnerable plaque and can help in identifying patients with high risk of cardiovascular events $[19,20]$. Increased MMP-9 expression in plaques with increased neovascular changes along with MRI scan of carotid arteries was studied but this analysis was performed in an animal study model.[23]

The aim of the present prospective pilot study is to analyse histological characteristics and expression of MMP-9 in carotid plaques of patients undergoing CEA and to investigate the correlation with preoperative clinical symptoms and MR features.

\section{Materials and methods}

\subsection{Study design and patients}

This pilot study is a prospective observational single-blind study of patients who underwent CEA at the Department of Cardiovascular Surgery in the Magdalena - Clinic for Cardiovascular Diseases and at the Department of Vascular Surgery in the University Hospital Merkur in Croatia over a 3-year study period. Institutional ethic committee approvals and Zagreb University School of Medicine ethic committee approval were obtained, and all patients gave their written informed consent. This study has been registered at the ISRCTN registry with study ID ISRCTN46536832. The study was conducted as per the Helsinki Declaration guidelines [24]. Identity of patients during data collection was anonymised and protected using a unique patient number.

Clinical history taking and physical examination including a neurological exam were performed by a surgeon. Clinical data including history of cardiovascular disease, risk factors for atherosclerosis, neurological event and timing since the most recent one, current medical treatment and computed tomography (CT) scans of brain were recorded. Stroke aetiology was determined based on a work-up including neurological examination, duplex ultrasound, MRI, CT scan of brain and echocardiography if required. Routine biochemical tests and highly sensitive C-reactive protein (hsCRP) were performed preoperatively. Before surgery, all patients underwent a duplex ultrasound plus MRI to grade internal carotid artery (ICA) stenosis and assess the supra-aortic and intracranial arterial system. The severity of carotid artery stenosis was defined using the North American Symptomatic Carotid Endarterectomy Trial Collaborators' (NASCET) criteria [25]. Inclusion criteria were that the patient should be already scheduled for CEA based on the indication for surgery with the consent to participate in the study. CEA was indicated according to the ESVS guidelines and recommendations of multicentric randomised controlled trials [1, 4, 25-27]. Exclusion criteria were contraindications to MRI (pacemaker, metallic implant, claustrophobia), presence of suboptimal MRI visualization of the carotid plaque or surgical specimen inadequate for histological or immunohistochemical analysis.

Patients were divided into two groups based on the presence of symptoms and the timing of the most recent symptom. Symptomatic patients were any patients who suffered a carotid stenosis territory symptom within the previous 6 months including ipsilateral stroke, transient ischaemic attack (TIA) or amaurosis fugax. Patients without any history of recent neurological symptoms or with nonspecific, non-hemispheric 
symptoms such as dizziness or vertigo were considered as asymptomatic.

\subsection{Preoperative magnetic resonance imaging}

MRI was performed using a 1.5-Tesla Vantage (Toshiba Medical Systems, Tokyo, Japan) or Avanto Scanner (Siemens, Erlangen, Germany) with a head and neck coil. Patient was placed in a standard dorsal position and the coil was combined with a head holder to stabilize position and prevent head rotation. A software syngo MR B19 2017 was used. A standardized MRI protocol was used to obtain four different contrastweighted images (time-of-flight [TOF] and T1-, PD- and T2-wighted) of the carotid arteries $2 \mathrm{~cm}$ proximal and $2 \mathrm{~cm}$ distal to the carotid bifurcation in axial and coronary planes [22, 28]. Fat suppression was used to reduce signal from subcutaneous fat tissue. MR angiography of carotid arteries was performed with 3 -dimensional TOF sequence (repetition time $[\mathrm{TR}] /$ time to echo $[\mathrm{TE}] /$ flip angle $[\mathrm{FA}]=30 \mathrm{~ms} / 8 \mathrm{~ms} / 20^{\circ}$; matrix 160x256 mm).

Each carotid lesion was classified using the modified American Heart Association (AHA) classification of carotid plaques identified by MRI [28]. The Modified AHA classification was derived by Cai et al. from experience in reviewing MR images and extensive literature review [28]. In this classification, we used: I-II) near-normal wall thickness with no calcification (AHA classification types I and II were merged into the type I-II due to a lack of the current resolution of MRI), III) diffuse intimal thickening or small eccentric plaque with no calcification, IV-V) plaque with a lipid or necrotic core surrounded by fibrous tissue with possible calcification (for a similar reason, AHA classification types IV and V were merged into the type IV-V), VI) complex plaque with possible surface defect, haemorrhage or thrombus, VII) calcified plaque, and VIII) fibrotic plaque without lipid core and with possible small calcifications. According to this modified classification, types IV-V and VI were classified as unstable plaques, while the others were classified as stable plaques. MRI criteria for the diagnosis of plaque vulnerability were thin fibrous cap, large lipid-rich or necrotic core, and plaque disruption or intra-plaque haemorrhage. MR images were stored in a digital archive as DICOM files and independently analysed by two experienced investigators blinded to the clinical history and histological findings. Any discrepancies were resolved by discussion between the two investigators.

\subsection{Histological analysis}

The carotid atherosclerotic plaque was excised during CEA using a standard surgical technique with minimal and atraumatic manipulation of the specimen previously described by Sef et al. [29]. The carotid plaque specimen was obtained immediately after the surgical excision and then stored in fresh $4 \%$ paraformaldehyde solution at $4^{\circ} \mathrm{C}$. The distal end of the plaque specimen was marked and processed by a standard procedure to obtain pathohistological specimen that includes fixation with $10 \%$ buffered formalin. The specimen was embedded in paraffin blocks, $4-\mu \mathrm{m}$-thick cross sections were dewaxed and prepared for subsequent staining.

The sections were stained with standard haematoxylin-eosin (H\&E) and with Masson method for detecting collagen tissue which will be semiquantitatively analysed according to the study by Verhoeven et al. [30]. Each specimen was cut into 5-mm-long sections from the central part of the plaque resulting in a total of 5 sections for histological analysis. Plaque specimens were analysed for lipid, fibrous tissue and calcium. Lipid core area was expressed as a percentage of the total plaque area (TPA) as described previously using the following semiquantitative scale: $0=<10 \%$ of TPA, $1=10-40 \%$ of TPA and $2=>40 \%$ of TPA $[31$, 32]. Histological examinations were performed independently by two experienced investigators blinded to the clinical history and MRI findings. Any discrepancies were resolved by discussion between the two investigators.

\subsection{Immunohistochemical analysis}

Immunohistochemical analysis of MMP-9 expression was performed in the central section of the plaque and one of the adjacent sections. Primary antibodies used in the study were: MMP-9 (Leica Mikrosysteme Vertieb GMBH, UK, monoclonal, clone 15W2, 1:50 dilution), CD68 PG-M1 (DakoCytomation, Denmark, monoclonal, clone PG-M1, RTU), smooth mucle actin (SMA) (DakoCytomation, Denmark, monoclonal, clone 1A4, RTU), CD34 (DakoCytomation, Denmark, monoclonal, clone QBend 10, RTU). 
Specimen sections were deparaffinized and antigens MMP-9, CD68 PG-M1, CD34 and SMA were unmasked in PT-module (DakoCytomation, Denmark) at 970C, 20 minutes in buffer derived from EnVision flex commercial "3 in 1" set (K8010, DakoCytomation, Denmark), and were then processed using a standardised automated procedure in an Autostainer device (DakoCytomation, Denmark), and stained with haematoxylin. Specimen sections were used as positive controls for the investigated biomarkers according to the manufacturer's recommendations. Semiquantitative analysis of macrophages and SMA by staining with CD68 PG-M1 antibody and SMA antibody, respectively, was performed according to the study by Verhoeven et al. [30]. Histological analysis of a type of bleeding was performed by determining the average density of small blood vessels by immunohistochemical staining of CD34 as described in the study by Derksen et al. [32]. The expression of MMP-9 protein was semiquantitatively analysed on specimen sections in macrophages and VSMC: $0=$ negative, $1=$ rare cells or one cluster of [?]20 cells, $2=$ less than 5 clusters of [?]20 cells, $3=[?] 5$ clusters consisted of [?]20 cells or one cluster [?]100 cells [33]. Analysis of endothelial cells was performed over 10 large areas with high magnification up to 400x, and the results were demonstrated as: $0=$ negative, $1=<25 \%, 2=25-50 \%, 3=>50-75 \%$ and $4=>75 \%$ of stained endothelial cells. The intensity of MMP-9 expression was demonstrated as: $0=$ negative, $1=$ visible using $400 x$ magnification, $2=100 x$ magnification and $3=40 \mathrm{x}$ magnification. Semiquantitative analysis of plaque specimen, immunohistochemical staining of macrophages, VSMC, microvascular density (MVD) and MMP-9 protein was performed using the software "ToupTekView" (Copyright (C), version x64 3.7.8481, www.touptek.com). Immunohistochemical examinations were performed independently by two experienced investigators blinded to the clinical history and MRI findings. Any discrepancies were resolved by discussion between the two investigators.

\subsection{Statistical analysis}

Data were collected prospectively. Categorical variables were tabulated as numbers with percentages. Continuous variables were shown as median values, with corresponding IQR. Analysis of the normal distribution of data (Kolmogorov-Smirnov test) was performed and, according to the results, appropriate non-parametric statistical analysis was performed. Differences among continuous variables were analysed using t-test and Mann-Whitney U test. Differences in categorical variables were analysed using Fisher's exact test. Difference between the groups was considered significant at the level of 0.05. Statistical analysis was performed using the licensed software IBM SPSS Statistica (version 25.0. www.statsoft.com, StatSoft, Inc. 2017).

\section{Results}

In this prospective pilot study, 15 patients with symptomatic (7 patients, $47 \%$ ) and asymptomatic carotid artery stenosis undergoing CEA were included. Among symptomatic patients, 5 (71\%) had recent stroke and $2(29 \%)$ had recent TIA with a median timing of the most recent event of 6 weeks (IQR: 1-18) before the surgery (Table 1). Demographic and clinical characteristics including risk factors, NASCET degree of stenosis, medications and preoperative laboratory results are summarized in Table 1. Symptomatic and asymptomatic patients did not significantly differ in respect to preoperative characteristics. Symptomatic patients had significantly higher hsCRP and D-dimer levels ( $p=0.016$ and $p=0.036$, respectively). Mean NASCET degree of stenosis was higher in the symptomatic as compared to the asymptomatic group (95\% [80-95] and $80 \%$ [76.3-83.8], $p=0.021$, respectively). There was no significant difference in the length of hospitalization and perioperative complications between the two groups, and only one patient in the symptomatic group had an episode of perioperative TIA which has recovered after the surgery.

Carotid plaque features detected on preoperative MRI scan are summarized in Tables 2 and 3. Prevalence of unstable plaque was higher in symptomatic than asymptomatic patients, although this was not statistically significant (63\% vs. $37 \%, p=0.077$ ) (Table 2, Fig. 1). The most common type of carotid plaque as per modified AHA classification was type IV-V (10 patients, 65\%) (Table 3).

Differences between preoperative symptoms and histological and immunohistochemistry features of carotid plaques are demonstrated in Table 4. The expression of MMP-9 in CD68 cells within the carotid plaque was found to be significantly higher in symptomatic as compared to asymptomatic patients ( $86 \%$ vs. $25 \%$ with the highest expression, $p=0.014)$. In the symptomatic group, 6 out of 7 plaque specimens had the 
expression of MMP-9 in [?]5 clusters consisted of [?]20 cells or one cluster [?]100 cells, while this was found only in one specimen in the asymptomatic group (Table 4, Fig. 2 and 3). It was also higher in SMA cells in symptomatic patients, but this did not reach statistical difference $(p=0.143)$. In addition, the average MVD by immunohistochemical staining of CD34 was higher among the symptomatic than asymptomatic patients (17 [6-23] vs. 6 [2.5-10], although this did not reach significance $(p=0.064)$. Similarly, lipid core area was larger among the symptomatic than asymptomatic patients, although it did not reach statistical significance $(p=0.132)$.

Differences between MRI features of carotid plaques and histological and immunohistochemistry features of carotid plaques are demonstrated in Table 5. There were no significant differences between several histological features and expression of MMP-9 between the patients with unstable or stable carotid plaque detected by preoperative MRI, although both the average MVD by immunohistochemical staining of CD34 and lipid core area were somewhat higher among patients with unstable than stable plaque. However, this was not significant ( $p=0.360$ and $p=0.569$, respectively) (Table 5).

\section{Discussion}

The atherosclerotic plaque is a dynamic lesion that undergoes continuous remodelling of the extracellular matrix on which its structural stability depends. Previous studies demonstrate that acute changes within the carotid plaque, such as IPH, fibrous cap rupture or ulceration can lead to the onset of ischemic cardiovascular events [6]. Recent reports suggest that MMP-9 may have a key role in all stages of the atherosclerotic process during which are being secreted by inflammatory cells and VSMC, although there is still lack of evidence whether MMP-9 can affect the incidence of adverse events [7, 8, 34].

The present pilot trial has demonstrated that the prevalence of unstable plaque was higher in symptomatic than asymptomatic patients, although this was not statistically significant ( $63 \%$ vs. $37 \%, p=0.077$ ). Our main findings were that the expression of MMP-9 in CD68 cells within the carotid plaque was found to be significantly higher in symptomatic as compared to asymptomatic patients $(86 \%$ vs. $25 \%$ with the highest expression, $p=0.014$ ). Importantly, the average MVD by immunohistochemical staining of CD34 was found to be higher and lipid core area larger among both symptomatic patients and unstable carotid plaque specimens, although this did not reach statistical significance $(p=0.064$ and $p=0.132, p=0.360$ and $p$ $=0.569$, respectively). Our study demonstrates the ability of MRI to identify patients with high-risk carotid plaque and to differentiate better which patients could benefit from early surgical revascularization. These findings are in line with the results of other studies and help in understanding that MRI can be the most accurate advanced imaging modality in distinguishing vulnerable from stable carotid plaques preoperatively, although further large prospective comparative studies are still required [7, 19, 20, 28, 35, 36]. Importantly, the most recent ESVS guidelines and recommendations of multicentric randomised controlled trials highlight clinical/imaging features in asymptomatic patients associated with an increased risk of stroke despite the best medical treatment that indicate necessity for carotid revascularisation [1, 4, 25-27]. A recent report comparing carotid MRI, ultrasound and histological findings of carotid plaque specimens has demonstrated that the findings of carotid MRI and ultrasound are closely associated with specimen morphology, but ultrasound alone is insufficient to detect the type of lesion accurately.[36] In addition, we have found that the most common type of carotid plaque as per modified AHA classification by MRI was type IV-V (10 patients, $65 \%$ ), while type VI was the most common (89 out of 252 plaque specimens) in the report by Cai et al.[28]. Similarly, Million et al. have reported the ability of MRI to identify high-risk carotid plaque and to differentiate symptomatic from asymptomatic patients, although their study was performed using a 3-Tesla system [19].

Furthermore, our results showed that MMP-9 may play a key role in remodelling and destabilization of the carotid plaque. As we hypothesized, the expression of MMP-9 in CD68 cells was significantly higher among symptomatic patients. Similarly, it was also higher in SMA cells in symptomatic patients, but this did not reach statistical difference $(p=0.143)$. Interestingly, symptomatic patients had significantly higher preoperative hsCRP which is in accordance with the inflammatory response and infiltration related to the destabilization of the plaque and potential increased release of MMP-9. Similarly, recent reports showed 
higher expression levels of MMP-9 obtained from symptomatic when compared to asymptomatic patients $[8,37,38]$. These findings highlighted MMP-9 as one of the most important enzymes related to the plaque instability. On the other hand, Baroncini et al. reported higher MMP-9 concentration in normal tissues and in asymptomatic patients suggesting that MMP-9 is a part of the atherosclerotic process although not associated to acute plaque disruption $[15,16]$. Still, this finding remains part of debate asHeo et al. have reported that MMP-9 is significantly associated with plaque rupture [38]. However, recent evidence suggests that an increase in proteolytic activity may lead to an overall increase in matrix degradation which could explain our results which demonstrate that the average MVD could be higher and lipid core area larger among both symptomatic patients and unstable carotid plaque specimens.

There are several limitations to our study. Our results showed a higher prevalence of unstable plaque in symptomatic patients, and a higher average MVD and larger lipid core area among both symptomatic patients and unstable carotid plaque specimens. However, the differences did not reach statistical significance probably because of the lack of power of the study and smaller sample size which are limitations of the present pilot study. Cappendijk et al. also failed to demonstrate a significant difference between symptomatic and asymptomatic patients [39]. However, in times of constraints on resources, both additional MRI analysis and detailed histologic work-up of plaques is costly and time-consuming, thus explaining the necessity of conducting a pilot trial. Although we obtained the best possible resolution for carotid MRI, another limitation of the study could be related to the use of only non-contrast MRI and motion artefacts. Further improvement in coil design, use of 3-Tesla system and modification of imaging parameters could be required, while the use of contrast-enhanced MRI would be less justified in terms of routine preoperative diagnostic work-up. Another limitation is that we have analysed our results semiquantitatively by immunohistochemistry, and the results do not allow accurate quantification. Nevertheless, our estimates were performed independently by two investigators. In addition, it is also possible that we have excluded some regions of plaques where inflammation was more advanced.

\section{Conclusions}

In this pilot study, we demonstrated that carotid MRI can be reliable in classifying carotid lesions and differentiating unstable from stable plaques. Our initial results showed that the expression of MMP-9 is significantly higher among symptomatic patients undergoing CEA which suggest that MMP-9 may affect remodelling and destabilization of the carotid plaque among symptomatic patients. This study could help in defining discriminative ability of preoperative MRI, and in improving identification of patients with unstable plaque that have an indication for CEA. However, further study will be anticipated with more participants being recruited over a longer study period. Further prospective and randomized studies are needed to provide better understanding of the mechanisms by which MMP-9 can affect atherosclerotic plaque structure and remodelling.

\section{Author contributions}

DS conceived and designed the research study, and prepared the original draft; DS, MM, MO, BJ, SK, AS, VV provided help on investigation, methodology, data collection, validation, and analyzed the data; MM performed statistical analysis; DS, MM, MO, TM, BJ, SK, AS, VV reviewed and edited the draft. All authors read and approved the final manuscript.

\section{Ethics approval and consent to participate}

All patients gave written informed consent, and relevant Institutional Review Boards approved this study.

\section{Acknowledgment}

We would like to express our gratitude to Dr. Juergen Falkensammer who helped with designing the research study. Support from Inel - Medical Technologies company by providing antibodies useful for this research project is greatly appreciated. Thanks to all the peer reviewers and editors for their opinions and suggestions.

\section{References}


[1] Naylor AR, Ricco JB, de Borst GJ, Debus S, de Haro J, Halliday A, et al . Editor's Choice - Management of Atherosclerotic Carotid and Vertebral Artery Disease: 2017 Clinical Practice Guidelines of the European Society for Vascular Surgery (ESVS). European journal of vascular and endovascular surgery : the official journal of the European Society for Vascular Surgery. 2018; 55: 3-81.

[2] Beneficial Effect of Carotid Endarterectomy in Symptomatic Patients with High-Grade Carotid Stenosis. 1991; 325: 445-53.

[3] Warlow C. MRC European Carotid Surgery Trial: interim results for symptomatic patients with severe (70-99\%) or with mild (0-29\%) carotid stenosis. The Lancet. 1991; 337: 1235-43.

[4] Halliday A, Mansfield A, Marro J, Peto C, Peto R, Potter J,et al . Prevention of disabling and fatal strokes by successful carotid endarterectomy in patients without recent neurological symptoms: randomised controlled trial. Lancet. 2004; 363: 1491-502.

[5] Peeters W, Hellings WE, de Kleijn DP, de Vries JP, Moll FL, Vink A, et al . Carotid atherosclerotic plaques stabilize after stroke: insights into the natural process of atherosclerotic plaque stabilization. Arteriosclerosis, thrombosis, and vascular biology. 2009; 29: 128-33.

[6] Redgrave JN, Gallagher P, Lovett JK, Rothwell PM. Critical cap thickness and rupture in symptomatic carotid plaques: the oxford plaque study. Stroke. 2008; 39: 1722-9.

[7] Olejarz W, Lacheta D, Kubiak-Tomaszewska G. Matrix Metalloproteinases as Biomarkers of Atherosclerotic Plaque Instability. International journal of molecular sciences. 2020; 21 (11).

[8] Loftus IM, Naylor AR, Goodall S, Crowther M, Jones L, Bell PR, et al . Increased matrix metalloproteinase-9 activity in unstable carotid plaques. A potential role in acute plaque disruption. Stroke. 2000; 31: 40-7.

[9] Loftus IM, Naylor AR, Bell PR, Thompson MM. Matrix metalloproteinases and atherosclerotic plaque instability. The British journal of surgery. 2002; 89: 680-94.

[10] Hermus L, Lefrandt JD, Tio RA, Breek JC, Zeebregts CJ. Carotid plaque formation and serum biomarkers. Atherosclerosis. 2010; 213: 21-9.

[11] Inokubo Y, Hanada H, Ishizaka H, Fukushi T, Kamada T, Okumura K. Plasma levels of matrix metalloproteinase-9 and tissue inhibitor of metalloproteinase-1 are increased in the coronary circulation in patients with acute coronary syndrome. American Heart Journal. 2001; 141: 211-7.

[12] Kai H, Ikeda H, Yasukawa H, Kai M, Seki Y, Kuwahara F, et al . Peripheral blood levels of matrix metalloproteases-2 and -9 are elevated in patients with acute coronary syndromes. Journal of the American College of Cardiology. 1998; 32: 368-72.

[13] Tang QD, Wu PS, Hou YQ, Huang Z, Zhou ZJ, Guo ZG, et al . [Plasma matrix metalloproteinases-2 and -9 levels are elevated in patients with acute coronary syndrome and coronary chronic total occlusion]. Nan fang yi ke da xue xue bao = Journal of Southern Medical University. 2009; 29: 1004-7.

[14] Loftus IM, Naylor AR, Bell PR, Thompson MM. Plasma MMP-9 - a marker of carotid plaque instability. European journal of vascular and endovascular surgery : the official journal of the European Society for Vascular Surgery. 2001; 21: 17-21.

[15] Baroncini LA, Filho Pazin A, Murta LO, Jr., Nakao LS, Ramos SG, Precoma DB. Videodensitometric analysis of advanced carotid plaque: correlation with MMP-9 and TIMP-1 expression. Cardiovascular Ultrasound. 2011; 9: 24.

[16] Baroncini VLA, Nakao LS, Ramos SG, Filho AP, Murta LO, Jr., Ingberman M, et al . Assessment of MMP-9, TIMP-1, and COX-2 in normal tissue and in advanced symptomatic and asymptomatic carotid plaques. Thrombosis journal.. 2011; 9: 6 . 
[17] Oates CP, Naylor AR, Hartshorne T, Charles SM, Fail T, Humphries K, et al . Joint recommendations for reporting carotid ultrasound investigations in the United Kingdom. European journal of vascular and endovascular surgery : the official journal of the European Society for Vascular Surgery. 2009; 37: 251-61.

[18] Gupta A, Baradaran H, Schweitzer AD, Kamel H, Pandya A, Delgado D, et al . Carotid plaque MRI and stroke risk: a systematic review and meta-analysis. Stroke. 2013; 44: 3071-7.

[19] Millon A, Mathevet JL, Boussel L, Faries PL, Fayad ZA, Douek PC, et al . High-resolution magnetic resonance imaging of carotid atherosclerosis identifies vulnerable carotid plaques. Journal of Vascular Surgery. 2013; 57: 1046-51 e2.

[20] Saito H, Kuroda S, Hirata K, Magota K, Shiga T, Tamaki N, et al . Validity of dual MRI and FFDG PET imaging in predicting vulnerable and inflamed carotid plaque. Cerebrovascular diseases (Basel, Switzerland). 2013; 35: 370-7.

[21] Wardlaw JM, Chappell FM, Stevenson M, De Nigris E, Thomas S, Gillard J, et al . Accurate, practical and cost-effective assessment of carotid stenosis in the UK. Health technology assessment. 2006; 10:iii-iv, ix-x, 1-182.

[22] Yuan C, Mitsumori LM, Ferguson MS, Polissar NL, Echelard D, Ortiz G, et al . In vivo accuracy of multispectral magnetic resonance imaging for identifying lipid-rich necrotic cores and intraplaque hemorrhage in advanced human carotid plaques. Circulation. 2001; 104: 2051-6.

[23] Jiang XB, Yuan WS, Wang JS, Liu Z, Liu DH, Shi ZS. Matrix metalloproteinase-9 expression in carotid atherosclerotic plaque and contrast-enhanced MRI in a swine model. Journal of neurointerventional surgery. 2014; 6: 24-8.

[24] World Medical Association Declaration of Helsinki: ethical principles for medical research involving human subjects. Jama. 2013; 310: 2191-4.

[25] Moneta GL, Edwards JM, Chitwood RW, Taylor LM, Jr., Lee RW, Cummings CA, et al. Correlation of North American Symptomatic Carotid Endarterectomy Trial (NASCET) angiographic definition of 70\% to $99 \%$ internal carotid artery stenosis with duplex scanning. Journal of Vascular Surgery. 1993; 17: 152-7; discussion 7-9.

[26] Randomised trial of endarterectomy for recently symptomatic carotid stenosis: final results of the MRC European Carotid Surgery Trial (ECST). Lancet. 1998; 351: 1379-87.

[27] Endarterectomy for asymptomatic carotid artery stenosis. Executive Committee for the Asymptomatic Carotid Atherosclerosis Study. JAMA. 1995; 273: 1421-8.

[28] Cai JM, Hatsukami TS, Ferguson MS, Small R, Polissar NL, Yuan C. Classification of human carotid atherosclerotic lesions with in vivo multicontrast magnetic resonance imaging. Circulation. 2002; 106: 1368-73.

[29] Sef D, Skopljanac-Macina A, Milosevic M, Skrtic A, Vidjak V. Cerebral Neuromonitoring during Carotid Endarterectomy and Impact of Contralateral Internal Carotid Occlusion. Journal of stroke and cerebrovascular diseases : the official journal of National Stroke Association. 2018; 27: 1395-402.

[30] Verhoeven BA, Velema E, Schoneveld AH, de Vries JP, de Bruin P, Seldenrijk CA, et al. Athero-express: differential atherosclerotic plaque expression of mRNA and protein in relation to cardiovascular events and patient characteristics. Rationale and design. European journal of epidemiology. 2004; 19: 1127-33.

[31] Hellings WE, Moll FL, de Vries JP, de Bruin P, de Kleijn DP, Pasterkamp G. Histological characterization of restenotic carotid plaques in relation to recurrence interval and clinical presentation: a cohort study. Stroke. 2008; 39: 1029-32.

[32] Derksen WJ, Peeters W, van Lammeren GW, Tersteeg C, de Vries JP, de Kleijn DP, et al . Different stages of intraplaque hemorrhage are associated with different plaque phenotypes: a large histopathological 
study in 794 carotid and 276 femoral endarterectomy specimens. Atherosclerosis. 2011; 218: 369-77.

[33] Welgus HG, Campbell EJ, Cury JD, Eisen AZ, Senior RM, Wilhelm SM, et al . Neutral metalloproteinases produced by human mononuclear phagocytes. Enzyme profile, regulation, and expression during cellular development. The Journal of clinical investigation. 1990; 86: 1496-502.

[34] de Nooijer R, Verkleij CJ, von der Thusen JH, Jukema JW, van der Wall EE, van Berkel TJ, et al . Lesional overexpression of matrix metalloproteinase- 9 promotes intraplaque hemorrhage in advanced lesions but not at earlier stages of atherogenesis. Arteriosclerosis, thrombosis, and vascular biology. 2006; 26: 340-6.

[35] Altaf N, Akwei S, Auer DP, MacSweeney ST, Lowe J. Magnetic resonance detected carotid plaque hemorrhage is associated with inflammatory features in symptomatic carotid plaques. Annals of vascular surgery. 2013; 27: 655-61.

[36] Arai D, Yamaguchi S, Murakami M, Nakakuki T, Fukuda S, Satoh-Asahara N, et al . Characteristics of carotid plaque findings on ultrasonography and black blood magnetic resonance imaging in comparison with pathological findings. Acta neurochirurgica. Supplement. 2011; 112: 15-9.

[37] Cipollone F, Prontera C, Pini B, Marini M, Fazia M, De Cesare D, et al . Overexpression of functionally coupled cyclooxygenase-2 and prostaglandin E synthase in symptomatic atherosclerotic plaques as a basis of prostaglandin $\mathrm{E}(2)$-dependent plaque instability. Circulation. 2001; 104: 921-7.

[38] Heo SH, Cho CH, Kim HO, Jo YH, Yoon KS, Lee JH, et al . Plaque rupture is a determinant of vascular events in carotid artery atherosclerotic disease: involvement of matrix metalloproteinases 2 and 9 . Journal of clinical neurology (Seoul, Korea). 2011; 7: 69-76.

[39] Cappendijk VC, Heeneman S, Kessels AG, Cleutjens KB, Schurink GW, Welten RJ, et al . Comparison of single-sequence T1w TFE MRI with multisequence MRI for the quantification of lipid-rich necrotic core in atherosclerotic plaque. Journal of magnetic resonance imaging : Journal of magnetic resonance imaging : JMRI. 2008; 27: 1347-55.

Tables

Table 1. Patients' characteristics

\begin{tabular}{llll}
\hline Characteristic & Symptomatic $(\mathbf{N}=7)$ & Asymptomatic $(\mathbf{N}=8)$ & $\mathrm{p}$ \\
\hline Age, years & $70(60,77 ; 48-77)$ & $69(64.3,72.8 ; 64-78)$ & 0.867 \\
Gender, male & $7(100)$ & $4(50)$ & 0.077 \\
Degree of Stenosis, \% & $95(80,95 ; 80-99)$ & $80(76.3,83.8 ; 70-85)$ & 0.021 \\
Timing of neurologic symptoms, weeks & $6(1,18 ; 1-20)$ & 0 & $<0.001$ \\
Diabetes mellitus & $4(57)$ & $3(38)$ & 0.619 \\
Arterial hypertension & $6(86)$ & $6(88)$ & 0.733 \\
Ischemic heart disease & $3(43)$ & $3(38)$ & 0.622 \\
Dyslipidaemia & $4(57)$ & $6(75)$ & 0.608 \\
COPD & $0(0)$ & $1(13)$ & 0.533 \\
Chronic kidney disease & $2(29)$ & $1(13)$ & 0.569 \\
Smoking, active & $3(43)$ & $1(13)$ & 0.386 \\
Smoking, previous & $0(0)$ & $2(25)$ & \\
Aspirin & $7(100)$ & $7(88)$ & 0.533 \\
Statins & $4(57)$ & $6(75)$ & 0.608 \\
Haemoglobin, g/L & $140(117,156 ; 114-162)$ & $136(128.3,142.8 ; 127-150)$ & 0.955 \\
Leukocyte count, x109/L & $8.7(7.3,10.9 ; 6.2-11)$ & $7.95(7,9.6 ; 5.3-11.5)$ & 0.336 \\
C-reactive protein & $13.3(5.2,27 ; 1.5-36.4)$ & $0.8(0.4,1.5 ; 0.4-1.8)$ & 0.016 \\
Serum glucose, mmol/L & $5.3(4.6,6.8 ; 4.4-6.8)$ & $5.45(5.3,8.5 ; 4.9-10.4)$ & 0.336 \\
PT & $0.805(0.74,0.91 ; 0.72-1)$ & $0.75(0.56,0.92 ; 0.56-0.92)$ & 0.548 \\
Fibrinogen, g/L & $5.65(3.0,7.5 ; 2.6-7.6)$ & 2.9 & 0.8
\end{tabular}




\begin{tabular}{llll}
\hline Characteristic & Symptomatic $(\mathbf{N}=\mathbf{7})$ & Asymptomatic $(\mathbf{N}=8)$ & $\mathrm{p}$ \\
\hline D-dimer, mg/L FEU & $0.87(0.61,1.19 ; 0.55-1.49)$ & $0.4(0.1,0.46 ; 0.1-0.46)$ & 0.036 \\
Total Cholesterol, mmol/L & $4.25(2.98,4.7 ; 2.6-4.8)$ & $3.65(2.55,4.45 ; 2.2-4.7)$ & 0.486 \\
LDL Cholesterol, mmol/L & $2.55(1.15 .3 .13 ; 0.7-3.3)$ & $2(1.1,3.1 ; 1.1-3.1)$ & 0.857 \\
HDL Cholesterol, mmol/L & $0.92(0.74,1.07 ; 0.7-1.09)$ & $0.91(0.9-1.2)$ & 0.629 \\
Triglycerides, mmol/L & $1.82(1.2,2.1 ; 1.04-2.2)$ & $0.8(0.54,1.29 ; 0.45-1.45)$ & 0.057 \\
Perioperative complications & $1(14)^{*}$ & $0(0)$ & 0.467 \\
Length of hospitalization, days & $6(3,6 ; 2-8)$ & $3(3,6 ; 2-6)$ & 0.281 \\
\hline
\end{tabular}

Data are median (quartiles; minimum-maximum) or count (percent). ${ }^{*}$ Transient ischaemic attack aPTT, activated partial thromboplastin time; ICA, internal carotid artery; FEU, fibrinogen equivalent units; HDL, high-density lipoprotein; LDL, low-density lipoprotein; PT, prothrombin time;

Table 2. MRI features of carotid plaques among symptomatic and asymptomatic patients

\begin{tabular}{llll}
\hline & MR features & MR features & \\
\hline & Stable & Unstable & $p^{\mathbf{1}}$ \\
Asymptomatic & $4(100)$ & $4(37)$ & 0.077 \\
Symptomatic & 0 & $7(63)$ & \\
\hline
\end{tabular}

Data are count (percent). ${ }^{1}$ Fisher's exact test

Table 3. MR features of carotid plaques by modified AHA classification.

\begin{tabular}{ll}
\hline AHA & $\mathbf{n ~ ( \% )}$ \\
\hline Type I-II: near normal thickness, no calcification & $0(0)$ \\
Type III: diffuse intimal thickening or small eccentric plaque with no calcification & $1(7)$ \\
Type IV-V: plaque with lipid or necrotic core surrounded by fibrous tissue with possible calcification & $10(65)$ \\
Type VI: complex plaque with possible disruption, haemorrhage or thrombus & $1(7)$ \\
Type VII: calcified plaque & $2(14)$ \\
Type VIII: fibrotic plaque without lipid core and with possible small calcifications & $1(7)$ \\
\hline
\end{tabular}

Data are count (percent).

Table 4. Differences between preoperative symptoms and histological and immunohistochemistry features of carotid plaques.

\begin{tabular}{lllll}
\hline & & Symptoms & Symptoms & $\mathrm{p}^{1}$ \\
\hline & & Asymptomatic (N=8) & Symptomatic $(\mathbf{N}=\mathbf{7})$ & $\mathbf{n}(\mathbf{\%})$ \\
MMP-9 CD34 & $\mathbf{n}(\mathbf{\%})$ & $1(14)$ & 0.273 \\
& No expression & $3(37.5)$ & $3(43)$ & \\
& $1-25 \%$ & $0(0)$ & $0(0)$ & \\
& $26-50 \%$ & $0(0)$ & $2(29)$ & $\mathbf{0 . 0 1 4}$ \\
& $51-75 \%$ & $2(25)$ & $1(14)$ & $1(14)$ \\
MMP-9 CD68 & $>75 \%$ & $3(37.5)$ & $0(0)$ &
\end{tabular}




\begin{tabular}{|c|c|c|c|c|}
\hline & & Symptoms & Symptoms & $\mathrm{p}^{1}$ \\
\hline & $<5$ clusters $>20$ cells & $2(25)$ & $0(0)$ & \\
\hline & $>5$ clusters $>20$ cells or 1 cluster $>100$ cells & $2(25)$ & $6(86)$ & \\
\hline \multirow[t]{4}{*}{ MMP-9 SMA } & No expression & $0(0)$ & $1(13)$ & 0.143 \\
\hline & Rare or 1 cluster $>20$ cells & $6(75)$ & $2(29)$ & \\
\hline & $<5$ clusters $>20$ cells & $0(0)$ & $2(29)$ & \\
\hline & $>5$ clusters $>20$ cells or 1 cluster $>100$ cells & $2(25)$ & $2(29)$ & \\
\hline MVD CD34 & n, median (interquartile ranges) & $6(2.5,10.0 ; 2,15)$ & $17(6,23 ; 1,34)$ & $0.064^{2}$ \\
\hline \multirow[t]{3}{*}{ lipid core/TPA } & $<10 \%$ & $0(0)$ & $0(0)$ & 0.132 \\
\hline & $10-40 \%$ & $6(75)$ & $2(29)$ & \\
\hline & $>40 \%$ & $2(25)$ & $5(71)$ & \\
\hline \multirow[t]{2}{*}{ SMA cells } & Low density and no circumference & $6(75)$ & $2(29)$ & 0.132 \\
\hline & High density and $100 \%$ circumference & $2(25)$ & $5(71)$ & \\
\hline \multirow[t]{2}{*}{ CD68 cells } & Low density & $2(25)$ & $1(14)$ & 1.000 \\
\hline & High density & $6(75)$ & $6(86)$ & \\
\hline
\end{tabular}

Data are count (percent) unless stated otherwise. ${ }^{1}$ Fisher's exact test. ${ }^{2}$ Mann Whitney test. MMP-9, matrix metalloproteinase 9; MVD, microvascular density; SMA, smooth muscle cells; TPA, total plaque area;

Table 5. Differences between MRI features of carotid plaques and histological and immunohistochemistry findings.

\begin{tabular}{|c|c|c|c|c|}
\hline & & MR features & MR features & $\mathrm{p}^{1}$ \\
\hline & & Stable & & \\
\hline \multirow[t]{2}{*}{$\begin{array}{l}(\mathrm{N}=4) \\
(\mathrm{N}=11)\end{array}$} & Unstable & & & \\
\hline & & n $(\%)$ & $\mathrm{n}(\%)$ & \\
\hline \multirow[t]{5}{*}{ MMP-9 CD34 } & No expression & $1(25)^{\prime}$ & $3(27)$ & 0.859 \\
\hline & $1-25 \%$ & $0(0)$ & $3(27)$ & \\
\hline & $26-50 \%$ & $0(0)$ & $0(0)$ & \\
\hline & $51-75 \%$ & $1(25)$ & $3(27)$ & \\
\hline & $>75 \%$ & $2(50)$ & $2(18)$ & \\
\hline \multirow[t]{4}{*}{ MMP-9 CD68 } & No expression & $0(0)$ & $1(9)$ & 1.000 \\
\hline & Rare or 1 cluster $>20$ cells & $1(25)$ & $3(27)$ & \\
\hline & $<5$ clusters $>20$ cells & $1(25)$ & $1(9)$ & \\
\hline & $>5$ clusters $>20$ cells or 1 cluster $>100$ cells & $2(50)$ & $6(55)$ & \\
\hline \multirow[t]{4}{*}{ MMP-9 SMA } & No expression & $0(0)$ & $1(9)$ & 0.671 \\
\hline & Rare or 1 cluster $>20$ cells & $2(50)$ & $6(55)$ & \\
\hline & $<5$ clusters $>20$ cells & $0(0)$ & $2(18)$ & \\
\hline & $>5$ clusters $>20$ cells or 1 cluster $>100$ cells & $2(50)$ & $2(18)$ & \\
\hline MVD CD34 & n, median (interquartile ranges) & $7(2,13.75 ; 2,15)$ & $10(5,19 ; 1,34)$ & $0.360^{2}$ \\
\hline \multirow[t]{3}{*}{ lipid core/TPA } & $<10 \%$ & $0(0)$ & $0(0)$ & 0.569 \\
\hline & $10-40 \%$ & $3(75)$ & $5(46)$ & \\
\hline & $>40 \%$ & $1(25)$ & $6(54)$ & \\
\hline \multirow[t]{2}{*}{ SMA cells } & Low density and no circumference & $2(50)$ & $6(54)$ & 1.000 \\
\hline & High density and $100 \%$ circumference & $2(50)$ & $5(46)$ & \\
\hline \multirow[t]{2}{*}{ CD68 cells } & Low density & $1(25)$ & $2(18)$ & 1.000 \\
\hline & High density & $3(75)$ & $9(82)$ & \\
\hline
\end{tabular}


Data are count (percent) unless stated otherwise. ${ }^{1}$ Fisher's exact test. ${ }^{2}$ Mann Whitney test. MMP-9, matrix metalloproteinase 9; MVD, microvascular density; SMA, smooth muscle cells; TPA, total plaque area;

\section{Figure legends}

Figure 1. Magnetic resonance imaging of unstable carotid plaque (modified AHA classification type VI) in a patient with symptomatic left internal carotid artery stenosis: A) and B) 3D-time of flight (TOF) sequences; C) Proton density (PD) sequence.

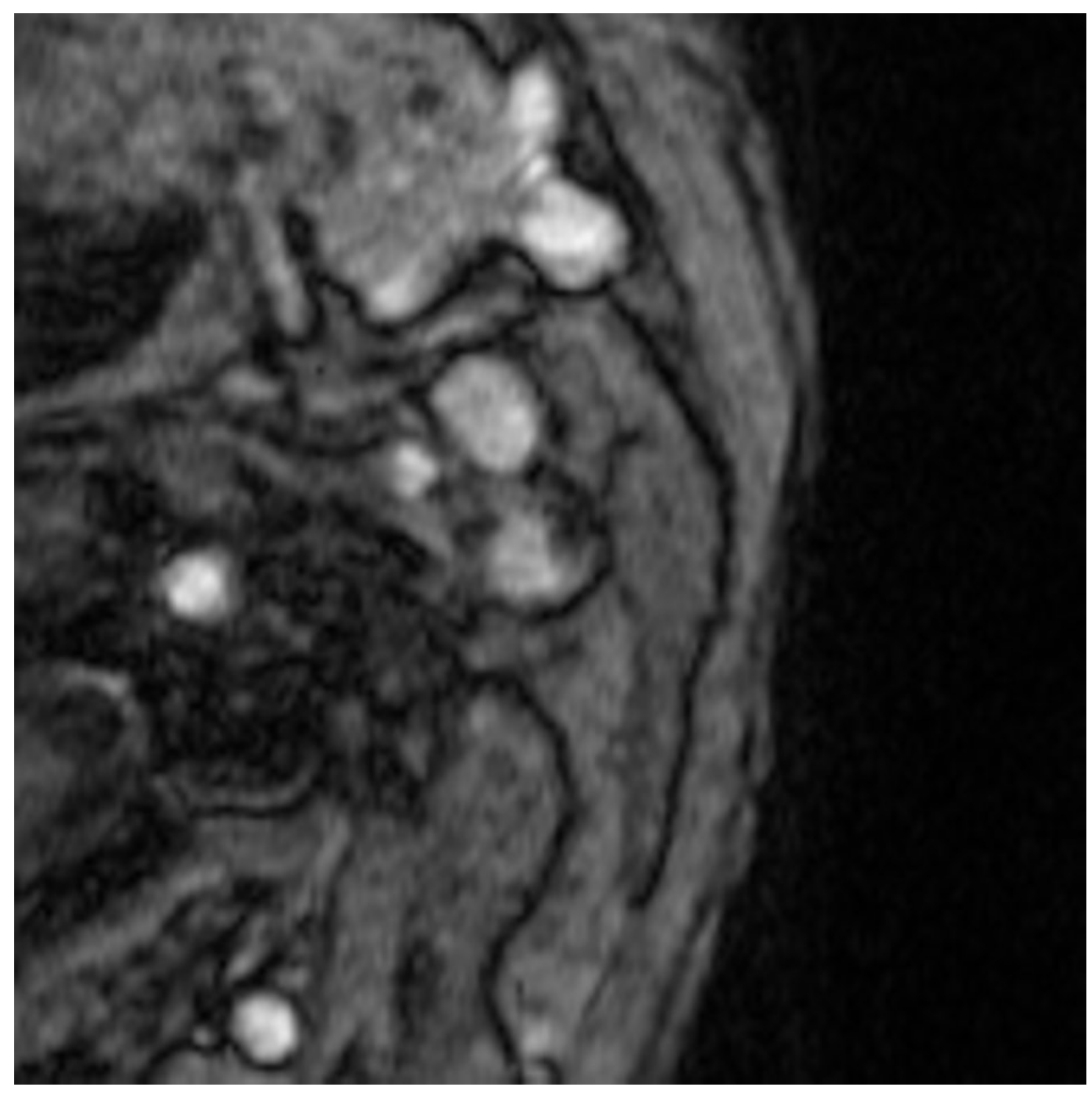

Figure 1: This is a caption

Figure 2. Immunohistochemical staining of MMP-9 positive cells in an unstable plaque on 400x magnification field of a patient with symptomatic carotid artery disease: A) MMP-9 positive endothelial cells, B) MMP-9 positive vascular smooth muscle cells, C) MMP-9 positive histiocytes. 


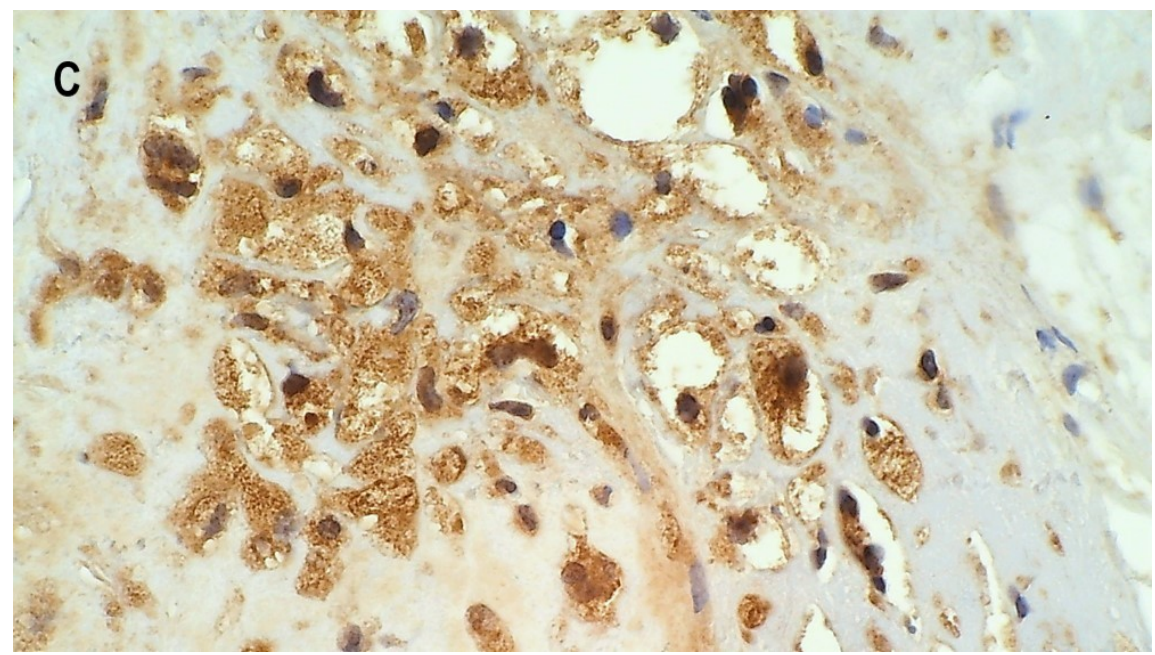

Figure 2: This is a caption

Figure 3. Immunohistochemical staining of MMP-9 negative cells in a stable plaque on 400x magnification field of a patient with asymptomatic carotid artery disease: A) MMP-9 negative endothelial cells, B) MMP-9 negative vascular smooth muscle cells, C) MMP-9 negative histiocytes.

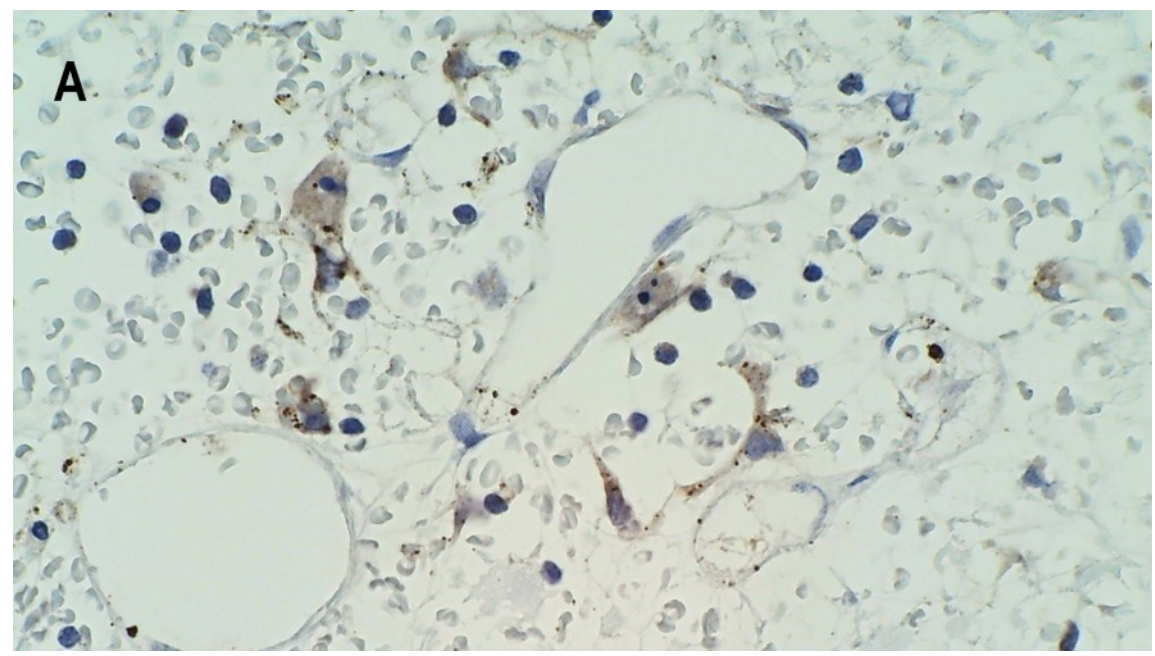

Figure 3: This is a caption 